\title{
Effect of filler and blending with SBR and NR on thermally induced crystallization of high-cis $B R$ as evaluated by dynamic mechanical analysis
}

\author{
M. Wunde, M. Klüppel* \\ Deutsches Institut für Kautschuktechnologie e. V., Eupener Straße 33, 30519 Hannover, Germany
}

Received 8 May 2019; accepted in revised form 2 September 2019

\begin{abstract}
The effect of thermally induced crystallization of high-cis polybutadiene (BR) on dynamic mechanical spectra is analyzed under different heating and cooling conditions. It is studied how the addition of carbon black and the blending with styrene-butadiene rubber (SBR) and natural rubber (NR) is affecting the crystallization. For unfilled BR an increased heating rate after fast cooling delivers less crystallization demonstrating that crystal formation requires time. With rising carbon black (CB) loading, a pronounced increase of crystallization speed is found indicating that CB supports nucleation and growth of BR crystals. In BR/SBR blends, which are detected as a single phase in dynamic mechanical spectra, crystallization is reduced for low SBR amounts and seems to disappear totally for 50/50 blends. In blends of BR, SBR, and NR the filler distribution is determined by comparing the peak heights in the loss modulus $G^{\prime \prime}$ of unfilled and filled compounds. The filler located in each phase is determined from the increase of the peak height corresponding to the respective phase. It is found that the filler is mainly located in the NR phase.
\end{abstract}

Keywords: rubber, dynamic mechanical analysis, thermal crystallization, high-cis polybutadiene, elastomer blends

\section{Introduction}

Thermally induced crystallization denotes the property of some polymers to crystallize in contrast to strain-induced crystallization without the need for straining. Some elastomers as polybutadiene (BR), natural rubber (NR), polyisoprene or polychloroprene, with regular composition show thermally induced crystallization [1]. This kind of crystallization is usually occurring at low temperatures but above the glass transition temperature $T_{\mathrm{g}}$. The crystallization speed can be expressed via the half-life $t_{1 / 2}$, the time, half of the crystallizable material needs to crystallize. The half-life strongly depends on the amount of regularly ordered monomers. For BR this is the cis- 1,4 content. BR with $97 \%$ cis- 1,4 content crystallizes more than 10 times faster than BR with $90 \%$ cis-1,4 [2]. Crystallization rates are depending on crosslinking as the crosslinks are hindering the diffusion of individual chain segments [1,2]. The crystallization half-life depends strongly on temperature. It is infinite for the glass transition temperature and the equilibrium melting temperature. The equilibrium melting temperature is the temperature at which an infinitely large solid and liquid have the same free energy. At higher temperatures, the free energy of the crystal is higher so that crystallization is not occurring. In between these temperatures, the logarithm of the half-life shows a parabolic dependence on temperature [1]. For BR the temperature of maximal crystallization rate $T_{\text {cryst }}$ is between -55 and $-56^{\circ} \mathrm{C}$ and does practically not depend on the microstructure [3]. For crosslinked BR between -70 and $-40^{\circ} \mathrm{C}$ a half-life around or smaller than $100 \mathrm{~s}$ is reported [1]. In this temperature interval, the time 
scale of crystallization is in the order of dynamic mechanical measurements. Melting of the crystallites depends on the crystallization temperature and occurs a few dozen degrees above the temperature the crystallites are formed [4]. This means that in dynamic mechanical measurements during heating crystallites start to form. At higher temperatures the formation of more stable crystallites and the melting of existing crystals occur simultaneously until finally all crystals are melted. The temperature process-dependent kinetics and the caloric behavior of crystallizable polymers can be simulated by a recently developed thermodynamic model [5]. This model shows that increasing the cooling or heating rate of measurement reduces the maximum level of crystallization.

Crystallization of elastomers can be observed e.g. by $\mathrm{X}$-ray diffraction, calorimetry and stress relaxation. Methods for crystalline studies are reviewed in [3]. BR crystallization is decreased by carbon black loading or by blending with SBR [6]. The larger the difference in $\delta$-parameter in blends of BR with nonpolar rubbers, the higher is the decrease of $\mathrm{BR}$ crystallization [7], leading to a smaller decrease of BR crystallization in a blend with SBR compared with the decrease in crystallization in a blend with NR [8]. The crystallization rate of NR is about three orders of magnitude smaller than for BR and therefore, for crosslinked NR, beyond our measuring times [1]. The increase of the storage and loss modulus in a temperature range where the crystallization speed is sufficiently fast can be seen as an effect of crystallization. Crystallization, also the partial crystallization found in the BR, increases the storage modulus as the material is more difficult to strain. This increase in storage modulus is decreasing when the crystallites melt. At least for small deformations, the decrease of storage modulus due to the melting is directly related to a peak in the loss modulus through the Kramers-Kronig relations.

The filler distribution can be evaluated by comparing the dynamic mechanical spectra of unfilled and filled immiscible elastomer blends [9-14]. The main idea is that filler increases the loss modulus peak of the respective polymer phase and so the filler distribution can be calculated from the increase of the different glass transition peaks. The height of the loss modulus peaks can thereby be influenced if one of the polymers is crystallizing during measurement. This is the case for blends with high-cis BR. High-cis BR is blended in tire tread compounds, especially to improve the rolling resistance of the tire.

This study is a supplement to previous works [13, 14] on the blend morphology and its influence on fracture mechanics within the collaboration in a fracture mechanical project. It has been found that the calculated filler distribution in NR/BR blends is affected by the crystallization of the BR [13]. The main idea for this study is to understand and possibly to subtract this effect. Therefore especially dynamic mechanical measurements have been done. A few differential scanning calorimetry measurements giving direct information on crystallization have been added. $\mathrm{X}$-ray diffracting as the standard method to investigate crystallization behavior is usually involving large synchrotron radiation facilities. Additionally, the filler distribution of triple blends is shown.

\section{Experimental}

\subsection{Materials}

The samples contain a high-cis butadiene rubber (Buna CB 24, Arlanxeo, Dormagen (Germany), $T_{\mathrm{g}} \approx$ $-109^{\circ} \mathrm{C}$ ) with min. $96 \%$ cis- 1,4 content. Some compounds are blends with styrene-butadiene rubber (Buna VSL 4526, Arlanxeo, Port Jérôme (France), $T_{\mathrm{g}} \approx-22^{\circ} \mathrm{C}$ ) and some of the blends also contain natural rubber (SVR CV 60, Vietnam, $T_{\mathrm{g}} \approx-60^{\circ} \mathrm{C}$ ). The compounds are unfilled or filled with up to $50 \mathrm{phr}$ carbon black N339 (Corax, Orion Engineered Carbons, Cologne (Germany)). The SBR is a solution styrenebutadiene rubber with $45 \mathrm{wt} \%$ vinyl and $26 \mathrm{wt} \%$ styrene groups. The samples are cross-linked semiefficiently by sulfur together with the vulcanization accelerator $N$-cyclohexyl-2-benzothiazole sulfonamide (CBS (Vulkacit CZ, Lanxess)). The samples were compounded with the processing and vulcanization additives stearic acid and $\mathrm{ZnO}$ (Rotsiegel, Grillo, Goslar (Germany)) and protected against aging by $N$-isopropyl- $N$-phenyl- $P$-phenylenediamine (IPPD (Vulkanox 4010, Lanxess)). The full recipes are shown in Table 1.

\subsection{Sample preparation}

The unfilled composites are mixed in a laboratory Haake mixer at $50 \mathrm{rpm}$ for 10 minutes, the filled compound for 20 minutes. After 5 minutes mastication of the polymers, the other ingredients are added. Leveling of the torque after mastication and after mixing indicates homogeneous mixtures. In mixtures with a 
Table 1. Recipes of the compounds.

\begin{tabular}{|l|c|c|c|c|c|c|c|c|c|}
\hline \multicolumn{1}{|c|}{ Samples } & BR & SBR & NR & CB & CBS & Sulfur & IPPD & ZnO & Stearic acid \\
\hline BR_0 & 100 & - & - & - & 2,5 & 1,7 & 1,5 & 3 & 1 \\
\hline BR_15 & 100 & - & - & 15 & 2,5 & 1,7 & 1,5 & 3 & 1 \\
\hline BR_30 & 100 & - & - & 30 & 2,5 & 1,7 & 1,5 & 3 & 1 \\
\hline BR_50 & 100 & - & - & 50 & 2,5 & 1,7 & 1,5 & 3 & 1 \\
\hline B90S10_0 & 90 & 10 & - & - & 2,5 & 1,7 & 1,5 & 3 & 1 \\
\hline B90S10_30 & 90 & 10 & - & 30 & 2,5 & 1,7 & 1,5 & 3 & 1 \\
\hline B90S10_50 & 90 & 10 & - & 50 & 2,5 & 1,7 & 1,5 & 3 & 1 \\
\hline B50S50_0 & 50 & 50 & - & - & 2,5 & 1,7 & 1,5 & 3 & 1 \\
\hline B50S50_30 & 50 & 50 & - & 30 & 2,5 & 1,7 & 1,5 & 3 & 1 \\
\hline B50S50_50 & 50 & 50 & - & 50 & 2,5 & 1,7 & 1,5 & 3 & 1 \\
\hline N55B23S23_0 & 22.5 & 22.5 & 55 & - & 2,5 & 1,7 & 1,5 & 3 & 1 \\
\hline N55B23S23_50 & 22.5 & 22.5 & 55 & 50 & 2,5 & 1,7 & 1,5 & 3 & 1 \\
\hline N70B15S15_0 & 15 & 15 & 70 & - & 2,5 & 1,7 & 1,5 & 3 & 1 \\
\hline N70B15S15_50 & 15 & 15 & 70 & 50 & 2,5 & 1,7 & 1,5 & 3 & 1 \\
\hline
\end{tabular}

higher amount of carbon black the rotor speed is lowered to keep the temperature below $120^{\circ} \mathrm{C}$. The curing system is added at $80 \%$ of the mixing time. Afterward, the compounds are handled on a roller mill. Vulcanization is performed at $150^{\circ} \mathrm{C}$ in a heat press up to $90 \%$ of the vulcameter torque maximum ( $t_{90}$ time).

\subsection{Dynamic mechanical analysis (DMA)}

The dynamic-mechanical measurements are carried out in the torsion-rectangular mode with a strip specimen of $2 \mathrm{~mm}$ thickness on the dynamic analyzer ARES (Rheometrix). This means that torsion is applied to a rectangular specimen. The width of the sample is $10 \mathrm{~mm}$ and the distance between the two clamps is around $30 \mathrm{~mm}$. The dynamic moduli are measured over a wide temperature range $\left(-115 \mathrm{C}\right.$ to $\left.+20^{\circ} \mathrm{C}\right)$ at a frequency of $1 \mathrm{~Hz}$ and $0.5 \%$ strain amplitude. All samples besides the unfilled BR are only measured

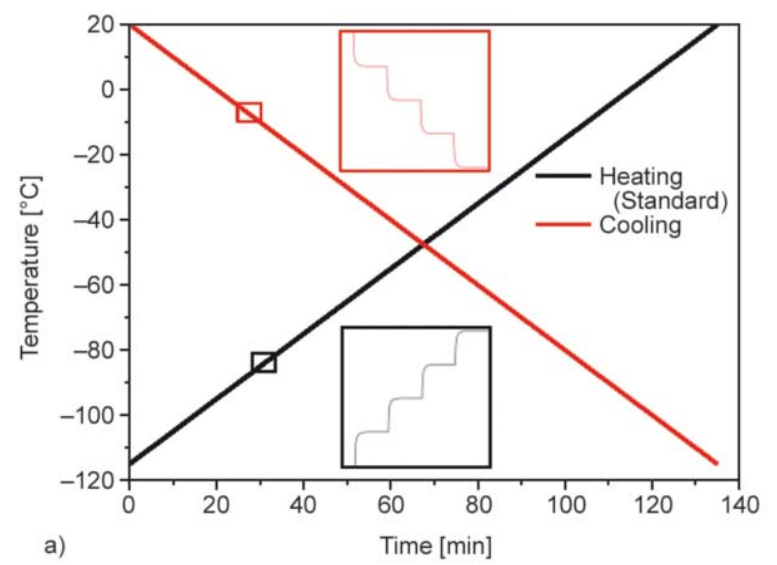

with the standard measurement procedure. In this procedure the sample is first quickly cooled to $-115^{\circ} \mathrm{C}$ (cooling rate $\sim 10^{\circ} \mathrm{C} /$ minute) and then measured while heating. In the heating process, the chamber temperature is increased successively by $1^{\circ} \mathrm{C}$. When this temperature is reached in the chamber it is kept for $45 \mathrm{~s}$ before storage and loss moduli are measured. This enables the sample to roughly adopt the temperature of the chamber. This procedure corresponds to a heating rate of around $1{ }^{\circ} \mathrm{C} /$ minute.

The unfilled BR is also measured with the cooling procedure. In this case, the chamber temperature is decreased successively by $1^{\circ}$, again with $45 \mathrm{~s}$ waiting time. In another measurement procedure, the chamber temperature is decreased and increased continuously with a cooling rate of $5{ }^{\circ} \mathrm{C} /$ minute and varying heating rates of 1,2 and $5^{\circ} \mathrm{C} /$ minute. The schematic temperature profiles in the chamber are shown in Figure 1.

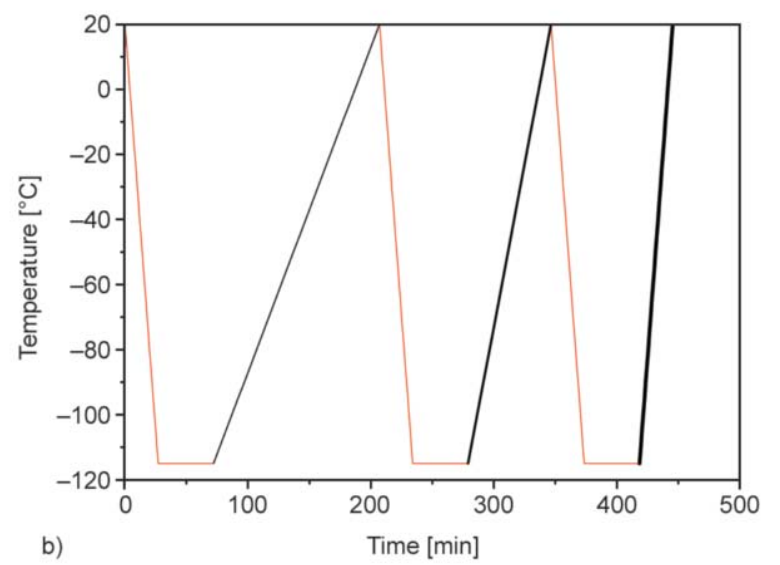

Figure 1. DMA-measurement procedures (schematic): Heating (Standard) and cooling measurement (a) and temperature ramp measurement (b). 


\subsection{Differential scanning calorimetry (DSC)}

The differential scanning calorimetry measurements were performed with a heat flux DSC 2920CE (TAInstruments) at a constant heating rate of $10^{\circ} \mathrm{C} / \mathrm{min}$ and sample weights of approximately $10 \mathrm{mg}$. The sample in a pan and an empty pan are quickly cooled by liquid nitrogen down to $-150^{\circ} \mathrm{C}$. During the following heating up to $100^{\circ} \mathrm{C}$, the heat fluxes between both pans and the machine are measured and subtracted to get the heat flow of the sample only.

\section{Results and discussion}

\subsection{Unfilled BR}

The effect of crystallization on the dynamic mechanical spectrum can be seen by comparing the measurement while heating after quick cooling (standard measurement procedure) with the same measurement while cooling the sample (Figure 2a). During the cooling the effect of crystallization can be seen in an increase of the storage modulus $G^{\prime}$ and the loss modulus $G^{\prime \prime}$ below $-40^{\circ} \mathrm{C}$. During the heating, the crystallization appears as broad peaks in the storage and loss modulus between -80 and $-20^{\circ} \mathrm{C}$. The local maximum of $G^{\prime}$ is found close to the temperature of maximal crystallization rate $T_{\text {cryst }} \approx-55^{\circ} \mathrm{C}$. For the heating (standard) measurement, representing crystallization at lower temperatures, smaller crystallites are expected than during cooling. Furthermore, recrystallization processes take place and lead to an increase in crystal size [15]. The non-equilibrium at measurement can be seen by a small shift of the glass transition temperature given by the maximum of the loss modulus.

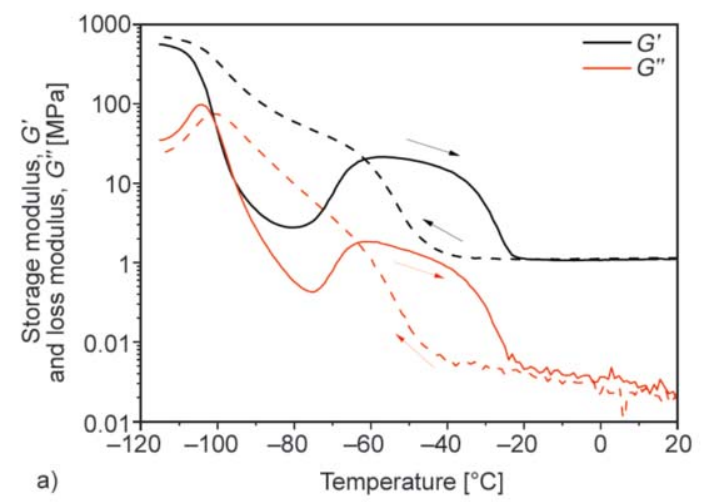

The effect of measurement duration can be seen in Figure $2 b$. This measurement was done with uniformly changing chamber temperatures at different rates of heating (temperature profile shown in Figure $1 \mathrm{~b}$ ). The heating rate depending shift of the glass transition can be seen at the global maximum of $G^{\prime \prime}(T)$ around $-100^{\circ} \mathrm{C}$. The crystallization can be observed in a second local maximum around the temperature of maximal crystallization $T_{\text {cryst }}$ of the BR. Due to the lower measuring times at the particular temperature interval, the crystallization observed as an increase of the moduli is decreasing with the heating rate. The form of the crystallization peak of $G^{\prime}$ might also give a hint whether the maximal crystallization speed is completed or not. In a 'round' peak (as in the $5^{\circ} \mathrm{C} /$ min curve) the most rapid crystallization is not completed when the increasing temperature starts to melt the crystallites. When in the slower measurements the most rapid crystallization has occurred, kind of plateaus with slightly decreasing values are formed. In this temperature range only some crystallization occurs but melting does not have started to a significant degree yet. The decrease of crystallization with increasing heating rate agrees with the expectations from thermodynamic considerations [5].

The crystallization behavior at the temperature of maximal crystallization rate $T_{\text {cryst }}$ seen in the dynamic mechanical analysis is displayed in Figure 3. In this measurement the sample is heated with a temperature ramp of $5{ }^{\circ} \mathrm{C} /$ minute from $-90{ }^{\circ} \mathrm{C}$ to $T_{\text {cryst }} \approx$ $-55.5^{\circ} \mathrm{C}$. At this temperature, the sample is kept for $10000 \mathrm{~s}$. In the heating period, the moduli are

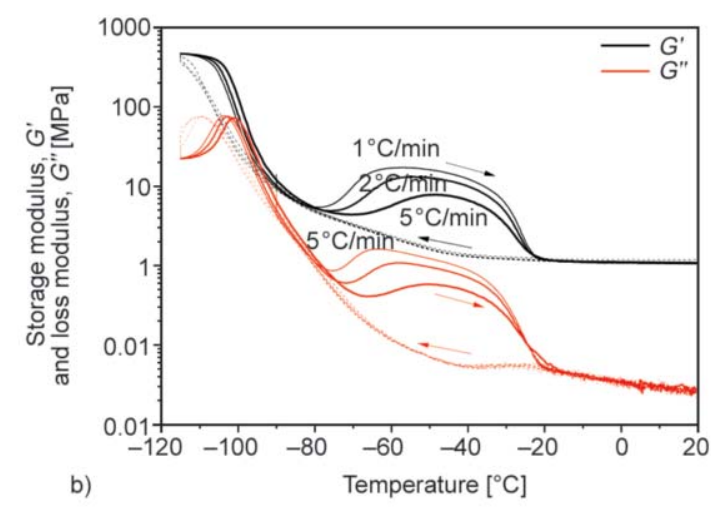

Figure 2. DMA-measurements of unfilled BR: Heating and cooling measurement where the heating is given as full lines and the cooling as dashed lines (a). Heating and cooling are stepwise according to Figure 1a with a heating rate of around $1{ }^{\circ} \mathrm{C} / \mathrm{min}$, for the heating measurement the sample was cooled down quickly before (cooling rate $\sim 10^{\circ} \mathrm{C} / \mathrm{min}$ ). Temperature ramp measurement according to Figure $1 \mathrm{~b}$ measuring different heating rates of 1,2 and $5^{\circ} \mathrm{C} / \mathrm{min}$ as indicated (b). The cooling is performed at a rate of $5^{\circ} \mathrm{C} / \mathrm{min}$. 


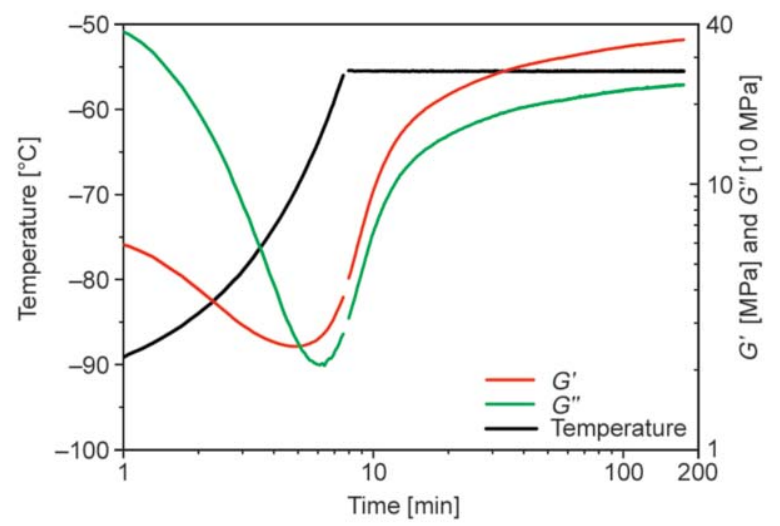

Figure 3. DMA-measurements of unfilled BR: Moduli measured during a temperature ramp of $5^{\circ} \mathrm{C} / \mathrm{min}$ from $-90^{\circ} \mathrm{C}$ to the temperature of maximal crystallization rate $T_{\text {cryst }} \approx-55.5^{\circ} \mathrm{C}$ and keeping this temperature for $10000 \mathrm{~s}$. Previously the compound was cooled quickly to $-90^{\circ} \mathrm{C}$.

primarily decreasing as the compound is departing from the glass transition. Closer to $T_{\text {cryst }}$ the moduli are increasing as the effect of crystallization dominates. The increase of the modulus is the strongest in the first minutes after reaching $T_{\text {cryst }}$. Afterward, the moduli are leveling out when the crystallization is nearly completed. It should be noted that in the first minutes of the measurement the sample temperature is not given by the chamber temperature and that no direct statements about the crystallization kinetics are possible from the increase of the moduli. Nevertheless, the time period until which most crystallization has appeared and which increase of the moduli this crystallization generates can be estimated.

\subsection{Filled BR}

The effect of carbon black on the crystallization seen in the dynamic mechanical spectra is shown in Figure 4. Again, fast cooling down to $-115^{\circ} \mathrm{C}$ was

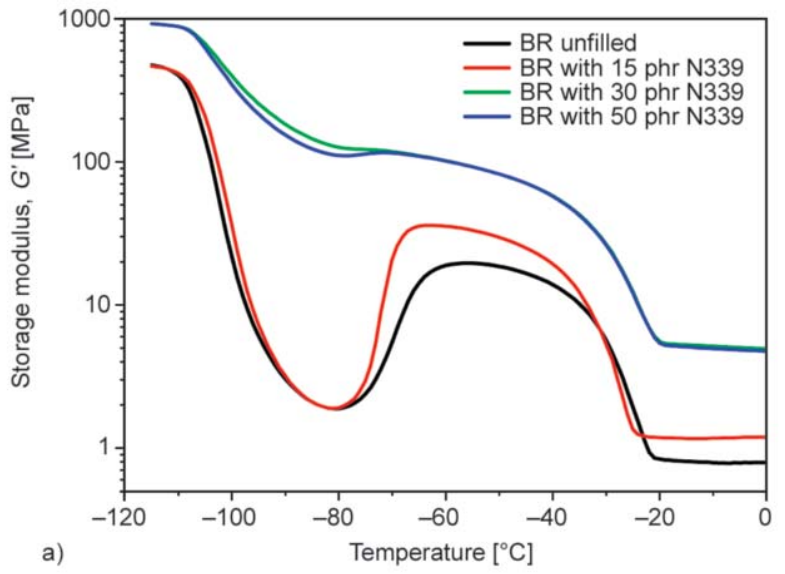

applied followed by heating according to Figure 1a. At moderate filler loading ( $15 \mathrm{phr}$ ) the crystallization peak in storage and loss modulus is enhanced by a factor of nearly two. This is more than expected by hydrodynamic amplification of the filler and indicates that a higher amount of crystallization is reached during the measurement. At higher filler loading the moduli at $T_{\text {cryst }} \approx-55^{\circ} \mathrm{C}$ reach values that seem to be nearly independent of filler loading. The moduli at this temperature are 5 or 6 times higher than those of the unfilled compound. This suggests that for $30 \mathrm{phr}$ an even higher degree of crystallinity is reached, that is not further increased by higher filler loadings. The reason for this could be that in these compounds the most pronounced increase of the moduli due to crystallization has already occurred at lower temperatures. For unfilled BR at $T_{\text {cryst }}$ the most pronounced increase happens within 20 minutes (see Figure 3), for the filled compounds it seems to be less. For the higher filler loadings also a pronounced increase of the modulus in the intermediate temperature range between glass transition and crystallization is seen. This indicates that the crystallization speed due to the presence of the filler increases substantially so that the crystallization already at temperatures of around $-90^{\circ} \mathrm{C}$ is sufficiently fast to yield the strongly increased moduli compared to the lower filled and unfilled sample. The crystallization is so fast that the moduli of the BR are not decreasing to ordinary values of rubbers above their glass transition $\left(G^{\prime}\right.$ of a few $\mathrm{MPa}$ ) before crystallization sets in. Instead for a long temperature interval intermediate values of $G^{\prime}$ around $100 \mathrm{MPa}$ are measured until the crystallites melt significantly. In all cases, crystallization plateaus are formed indicating that a higher portion of final crystallization degree is reached. These results seem

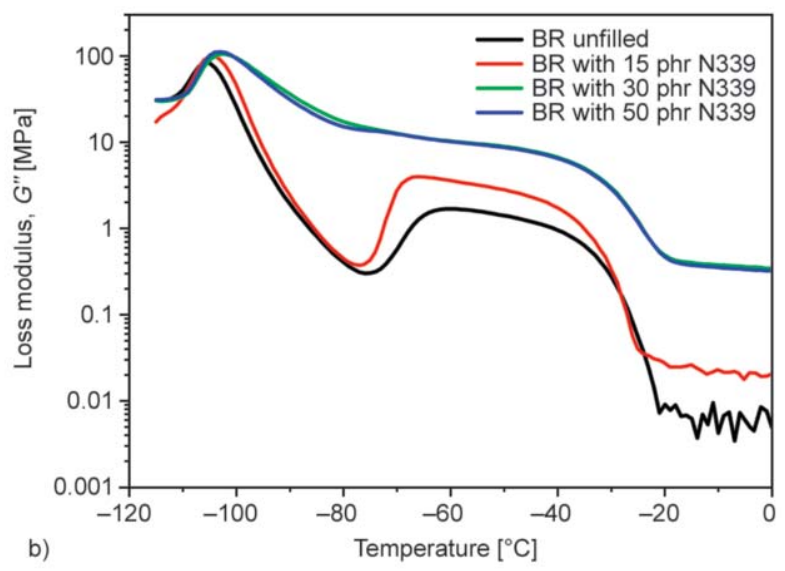

Figure 4. DMA-measurements of BR filled with different amount of carbon black: Storage modulus (a) and loss modulus (b). 


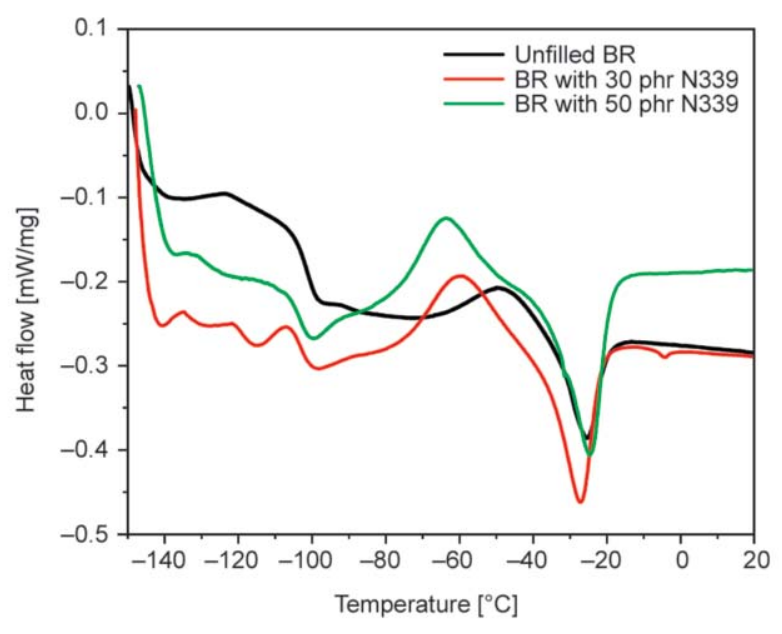

Figure 5. Heat flows measured by DSC of unfilled BR and filled BR with 30 and $50 \mathrm{phr}$ carbon black.

to contradict previous findings where the carbon black content decreases BR crystallization [5]. The main reason for that discrepancy is that from the analysis of the moduli the crystallization speed can be compared, not the level of final crystallization. A possible explanation of the increased moduli in filled BR could be synergetic reinforcement of filler and crystallites that lead to higher moduli, although less crystallization could be present compared to the unfilled BR. Another possibility could be increased nucleation due to the presence of carbon black.

Figure 5 shows DSC measurements of the unfilled and filled BR compounds. The glass transition can be seen as a step-like decrease of heat flow for all samples at around $-100^{\circ} \mathrm{C}$. The unfilled sample starts to crystallize at around $-70^{\circ} \mathrm{C}$ and crystallization reaches a maximum at around $-50^{\circ} \mathrm{C}$, indicated by the flat peak of the heat flow. Contrary, the filled compounds are starting to crystallize directly after the glass transition and the maximum crystallization is reached earlier at around $-65^{\circ} \mathrm{C}$. All compounds are melting at around $-25^{\circ} \mathrm{C}$. These observations are in line with the DMA measurements and confirm the results and interpretations of Figure 4.

\subsection{BR/SBR blends}

Mixing entropy is very small for polymers because of the small number of molecules involved due to their high molecular weight. Therefore most polymer blends are not miscible. The miscibility of two polymers can be predicted by the comparison of their solubility parameters $\delta$. The $\delta$-parameter is defined as the square root of the energy of vaporization per volume and is related to the cohesion energy between identical monomers. Only for very small differences of $\delta$, it is expected that the two polymers form a common phase. In that case, only one glass transition in between both polymer glass transitions occurs. Already for small differences of the solubility parameter, the very low mixing entropy of long polymer chains is surmounted so that the phases separate. A very broad glass transition indicates micro phases with different content of the two polymers [16-18].

The miscibility of BR and SBR depends on the composition of the polymers. Cis 1,4-polybutadiene, as the polymer with the least voluminous side groups, has the lowest glass transition temperature and both, the addition of vinyl and styrene units is increasing the volume of side groups and therefore increasing $T_{\mathrm{g}}$. But the solubility parameter which is connected to the vaporization energy is increasing linearly with the styrene content and decreasing linearly with the vinyl content [19]. The SBR used here has a high vinyl content of $45 \%$ which nearly counterbalances the decrease in $\delta$ due to the styrene content of $26 \%$. This makes the BR and the SBR used in this study at least partially miscible. The storage and loss moduli of the BR/SBR blends are shown in Figure 6. The glass transition is seen in a single broad peak in the loss modulus $G^{\prime \prime}$ indicating a global mixture with locally varying polymer concentrations. Such a mixture we will call in the following a mixed phase. In a 50:50 blend the BR and the SBR form a mixed phase which can be seen as broad peak of the loss modulus in between the glass transition temperatures of the polymers but closer to the glass transition of the BR (Figure 6d) $\left(T_{\mathrm{g}} \sim-86^{\circ} \mathrm{C}\right)$. Crystallization can be seen neither in the storage modulus (Figure 6c) nor in the loss modulus, even not for the filled compounds. Nevertheless, a low amount of crystallization which is not directly visible in DMA is possible. For a 90:10 blend the glass transition peak in the loss modulus shifts due to the higher amount of BR to lower temperatures close to $-100^{\circ} \mathrm{C}$ (Figure $\left.6 \mathrm{~b}\right)\left(T_{\mathrm{g}} \sim-102^{\circ} \mathrm{C}\right)$. A crystallization peak is visible in storage modulus (Figure 6) and loss modulus. These peaks are increasing with filler volume fraction. The crystallization rate is clearly smaller compared to the pure BR. This can be seen in lower local maxima around $T_{\text {cryst }}$ and in more curved shape of these peaks indicating that the most rapid crystallization is not finished. The results agree with the known hindrance of SBR on BR crystallization [6-8]. 

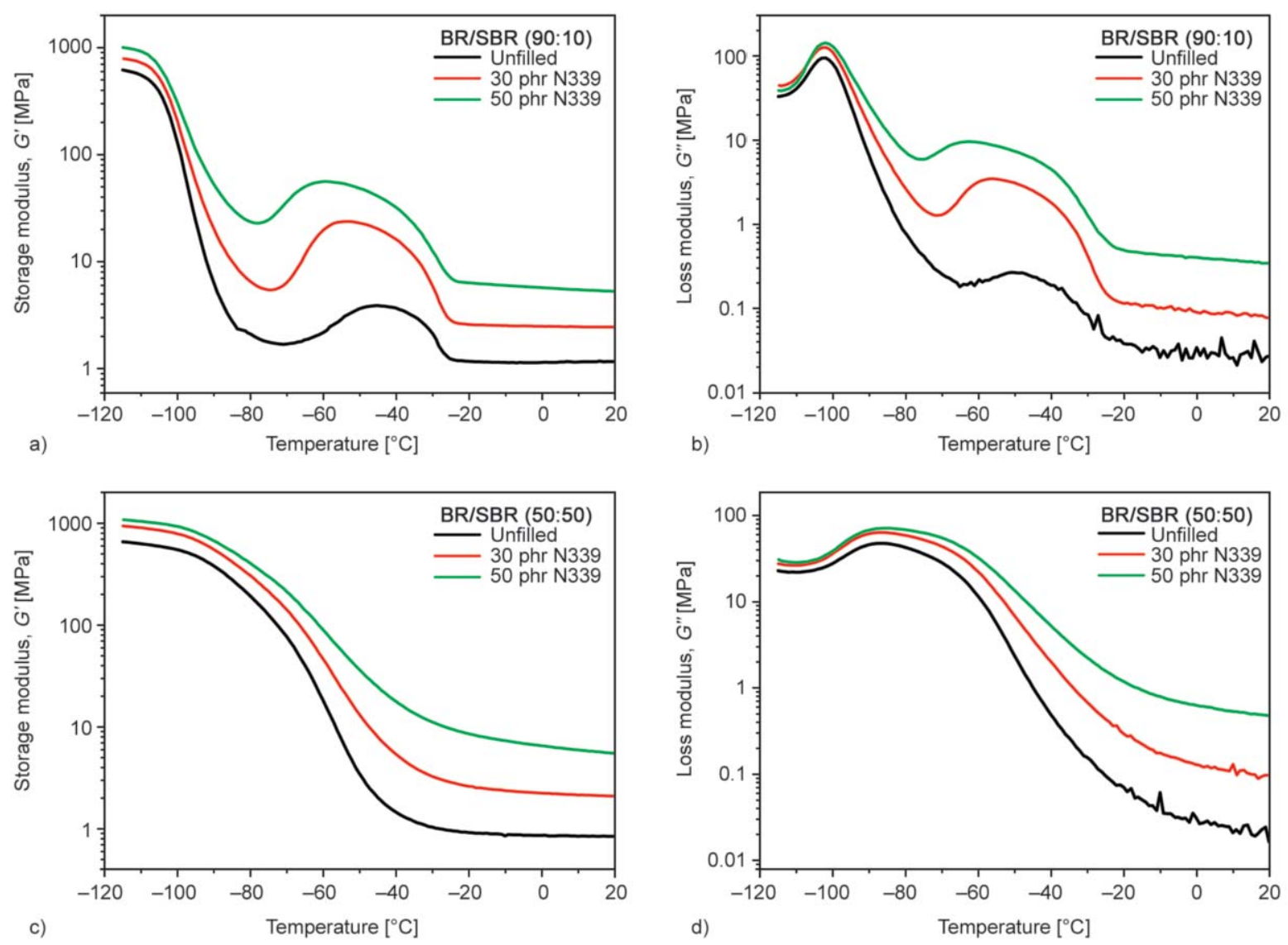

Figure 6. DMA-measurements of BR/SBR blends with different blend ratio and amount of carbon black: Storage modulus $G^{\prime}$ (a) and loss modulus $G^{\prime \prime}$ (b) of (90:10) blend, storage modulus $G^{\prime}$ (c) and loss modulus $G^{\prime \prime}$ (d) of (50:50) blend.

\subsection{NR/BR/SBR blends}

$\mathrm{NR}$ is miscible neither with the BR nor with the SBR. In the NR/BR/SBR blends two glass transitions can be observed (Figures 7 and 8): The glass transition of the NR is at around $-60^{\circ} \mathrm{C}$ and the glass transition of the BR/SBR mixed phase at lower temperatures. The loss modulus $G^{\prime \prime}$ of the mixed BR/ SBR phase, as discussed in the previous chapter, is maximal between -80 and $-90^{\circ} \mathrm{C}$ or around $-100^{\circ} \mathrm{C}$, depending on the composition of the mixed BR/SBR phase (compare with Figure 6). A possible crystallization especially of the compounds with high BR content is not visible as the glass transition of the NR around $-60^{\circ} \mathrm{C}$ is hiding possible effects of BR crystallization occurring in a similar temperature range.
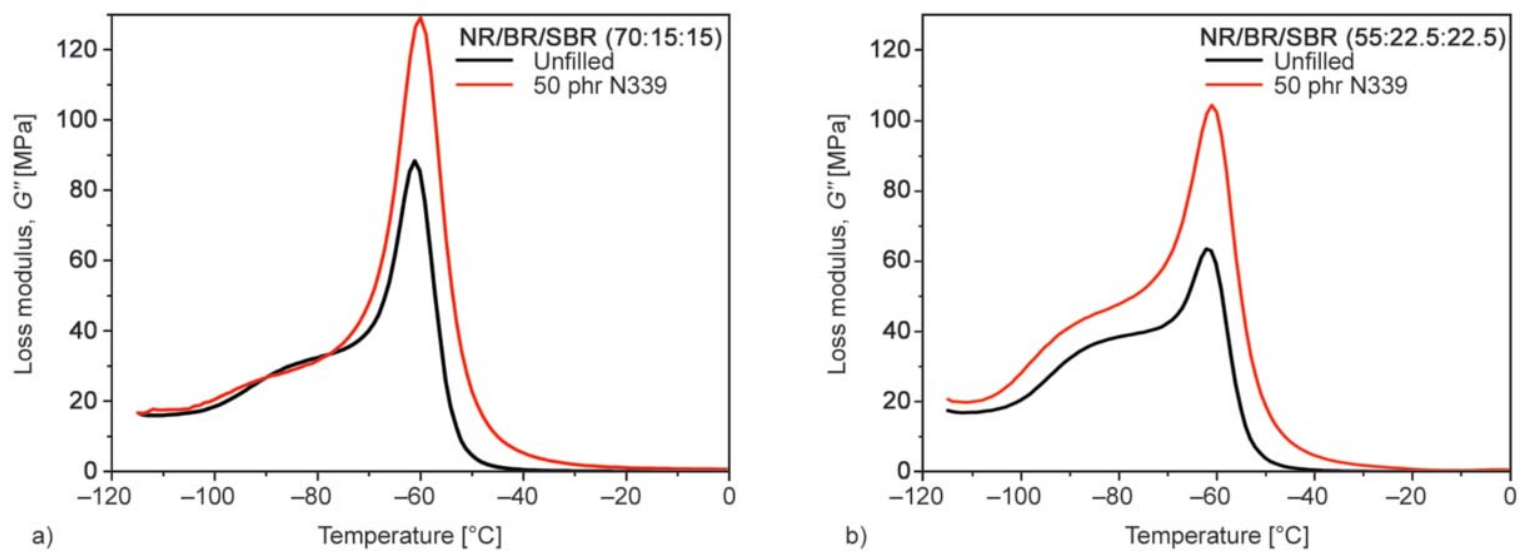

Figure 7. Loss modulus $G^{\prime \prime}$ of NR/BR/SBR blends (equal amount of BR and SBR): Unfilled and filled blends with 70 phr NR (a) and with 55 phr NR (b). 

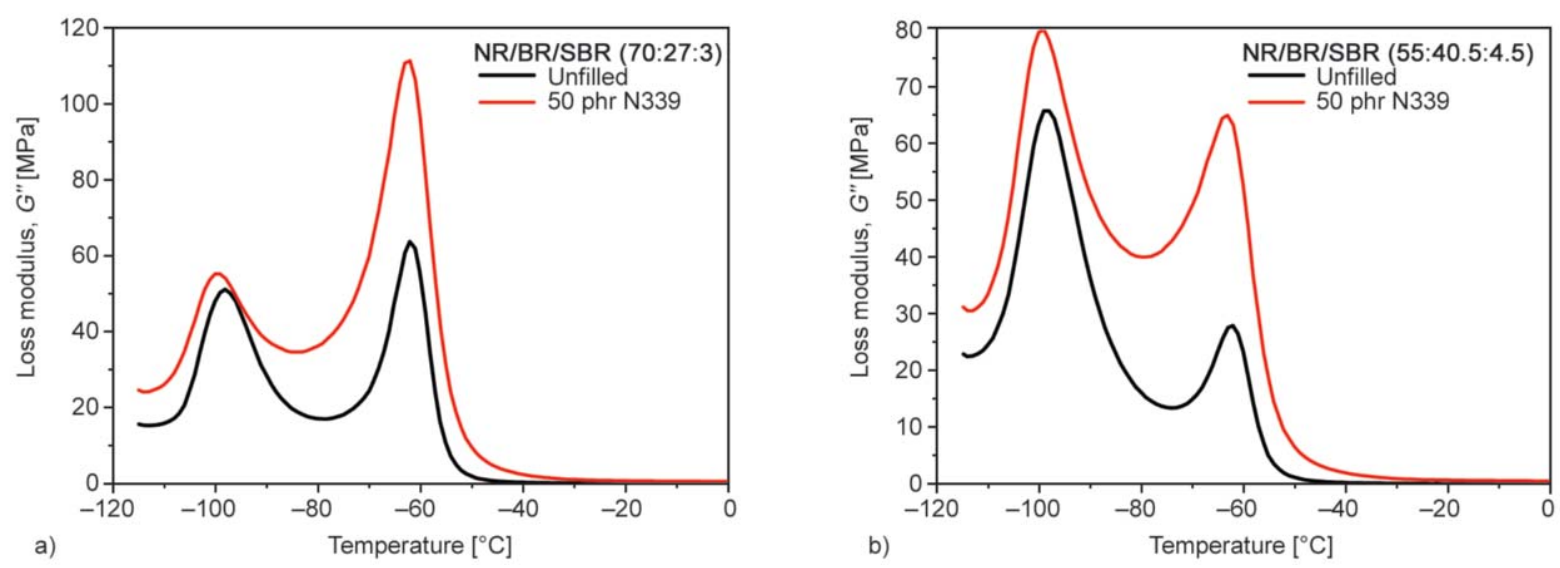

Figure 8. Loss modulus $G^{\prime \prime}$ of NR/BR/SBR blends (very small amount of SBR): Unfilled and filled blends with 70 phr NR (a) and with $55 \mathrm{phr}$ NR (b).

\subsection{Filler distribution in $\mathrm{NR} / \mathrm{BR} / \mathrm{SBR}$ blends} The dissipated energy $\Delta E$ of a harmonic strained rubber with amplitude $\gamma_{0}$ is directly related to the loss modulus $G^{\prime \prime}$ [9], as shown by Equation (1):

$$
\Delta E=\pi G^{\prime \prime} \gamma_{0}^{2}
$$

Due to the additivity of dissipated energy the dissipated energy in a polymer blend $\Delta E_{\text {blend }}$ equals the sum of the dissipated energies $\Delta E_{\mathrm{i}}$ in the different polymer phases $i$. According to the different stiffness, the phases are strained with their specific amplitude $\gamma_{0, \mathrm{i}}$ see Equation (2):

$$
\Delta E_{\text {blend }}=\sum_{\mathrm{i}} \Delta E_{\mathrm{i}}=\sum_{\mathrm{i}} \pi \phi_{\mathrm{i}} G_{\mathrm{P}, \mathrm{i}}^{\prime \prime} \gamma_{0, \mathrm{i}}^{2}
$$

where, $\phi i$ and $G^{\prime \prime}{ }_{\mathrm{P}, \mathrm{i}}$ are the volume fraction and the loss modulus of the $i$-th phase, respectively. For the loss modulus of the blend see Equation (3):

$$
G_{\text {blend }}^{\prime \prime}=\sum_{\mathrm{i}} G_{\mathrm{P}, \mathrm{i}}^{\prime \prime} \phi_{\mathrm{i}} \frac{\gamma_{0, \mathrm{i}}^{2}}{\gamma_{0, \mathrm{i}}^{2}}
$$

We consider a polymer blend with different phases and sufficiently separated glass transition temperatures of the phases. In the temperature range just below the glass transition temperature of the higher $T_{\mathrm{g}}$ phase this phase is in a glassy state and exhibits a high stiffness while all other phases are soft. In ref. [9] the mechanical response of the glassy phase network was modeled as a percolation network: Below a critical concentration $\phi_{\mathrm{c}}$ the glassy polymer phase builds a hard dispersed phase with spatially separated domains within one or more soft continuous phases. In this case, the glassy polymer phase increases the stiffness of the composite only by hydrodynamic amplification. At the critical concentration $\phi_{\mathrm{c}}$ the separated glassy domains form a co-continuous phase network which is dominating the elastic modulus at concentrations $\phi_{\mathrm{i}}>\phi_{\mathrm{c}}$. In a percolation network, only the singly connected links which are not building close loops are deformed under external strain. Therefore, the elastic modulus of the composite is strongly affected by the distribution of singly connected links. This also determines the deformation $\gamma_{0, \mathrm{i}}$ of the glassy phase which shows power-law behavior above the percolation threshold $\phi_{\mathrm{c}}$. The expression $\phi_{\mathrm{i}}\left(\gamma_{0, \mathrm{i}}^{2} / \gamma_{0}^{2}\right)$ in Equation (3) is the amplitude height of the polymer phase in an unfilled blend compared to the pure polymer and can be written as power-law increase with elasticity exponent $\tau$ if only one phase is in the glassy state [9], as shown by Equation (4):

$\phi_{\mathrm{i}} \frac{\gamma_{0, \mathrm{i}}^{2}}{\gamma_{0, \mathrm{i}}^{2}}=\left(\frac{\phi_{\mathrm{i}}-\phi_{\mathrm{c}}}{1-\phi_{\mathrm{c}}}\right)^{\tau}$

Lower values than the theoretical value of $\tau \approx 3.6$ were found and explained by coalescence phenomena on small length scales [9].

Semi-empirically the term $\phi_{\mathrm{i}}\left(\gamma_{0, \mathrm{i}}^{2} / \gamma_{0}^{2}\right)$ in Equation (3) can also be fitted by quadratic equations, so that the loss modulus of the blend $G^{\prime \prime}$ blend is given by Equation (5) [12]:

$$
G_{\text {blend }}^{\prime \prime}=\sum_{\mathrm{i}} G_{\mathrm{P}, \mathrm{i}}^{\prime \prime}\left(a_{\mathrm{i}}+b_{\mathrm{i}} \phi_{\mathrm{i}}+c_{\mathrm{i}} \phi_{\mathrm{i}}^{2}\right)
$$

The lower branch is adopted by the polymer with the higher glass transition temperature $T_{\mathrm{g}}$ and the higher branch by the polymer with the lower $T_{\mathrm{g}}$. In Figure 9 these ratios are shown for the unfilled NR/BR/SBR blends. In this case, the NR is found on the lower branch and the BR/SBR mixed phase on the upper branch. For lower contents of BR and SBR in the blend the evaluation becomes difficult as the broad BR/SBR peak gets covered by the low-temperature 


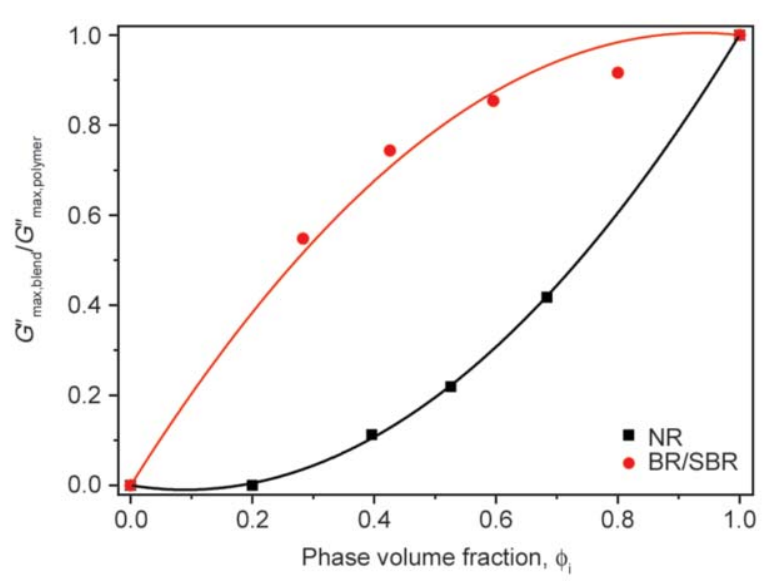

Figure 9. Amplitude height $G^{\prime \prime}$ max,blend of a polymer phase in an unfilled blend compared to the amplitude height

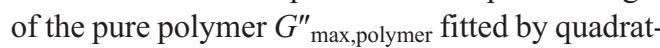
ic equations.

tail of the NR. The quadratic function for the NR phase of the NR/BR/SBR blends is quite close to the quadratic function found for the NR phase in NR/BR blends [13].

In this method, it is assumed that the loss modulus $G^{\prime \prime}$ of the compound is the sum of the loss moduli of the different polymer phases. In fact, the complex modulus is formed in different ways that dependent on the blend morphology. There are quite different results obtained, e.g. when the phases are ordered in parallel or in series. For the case of spherical inclusions in a matrix, the Olroyd-Palierne model [20] or self-consistent models [21] can be used. For most blend ratios studied here, the different phases build separate continuous networks for which these models cannot be used.

Neglecting hydrodynamic effects of the filler particles the loss modulus of a filled polymer at the glass transition temperature $G^{\prime \prime}\left(T_{\mathrm{g}}\right)$ is expected to

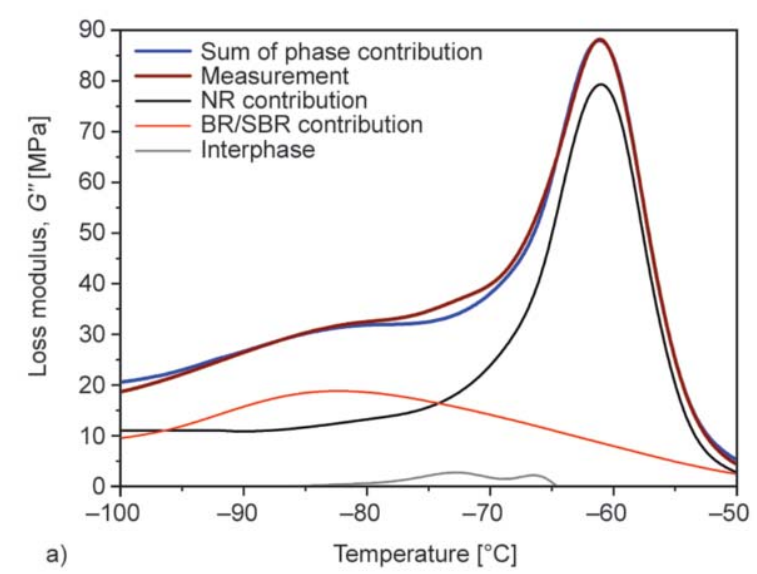

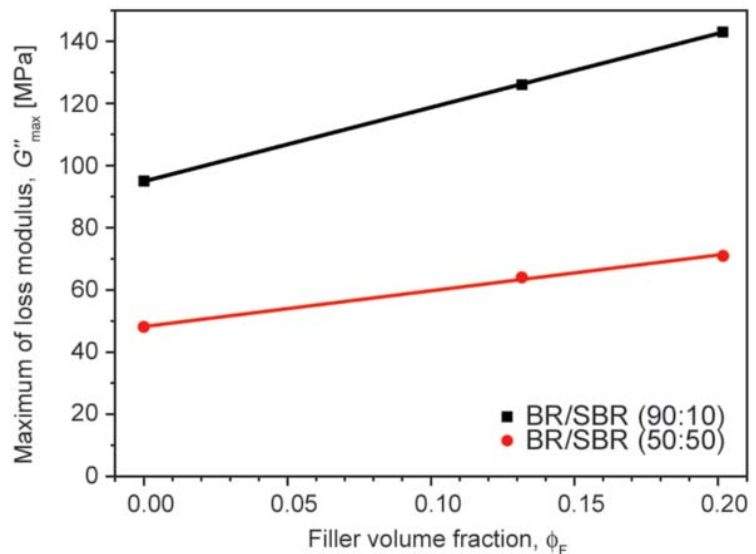

Figure 10. Linear increase of the maximum of loss modulus $G^{\prime \prime}$ (glass transition of BR/SBR weights) with filler volume fraction.

be linearly increasing with filler volume fraction $\phi_{\mathrm{F}}$, see Equation (6) [9]:

$G^{\prime \prime}\left(T_{\mathrm{g}}\right)=G^{\prime \prime}{ }_{0}\left(T_{\mathrm{g}}\right)+\alpha \phi_{\mathrm{F}}$

where, $G^{\prime \prime}{ }_{0}\left(T_{\mathrm{g}}\right)$ is the loss modulus of the unfilled polymer at the glass transition temperature and $\alpha$ is the slope of loss modulus with filler volume fraction. In fact, this linear behavior is found for a variety of polymer blends [9-12]. Linear increase with filler volume fraction was also already found for all three polymer compounds used here [13-14].

The maximum loss modulus $G^{\prime \prime}\left(T_{\mathrm{g}}\right)$ of the filled compounds can also be measured for the mixed BR/SBR phase. Also, the maximum of the mixed phase is increasing linearly with the filler volume fraction (Figure 10).

Combining the effect of the filler (Equation (4)) with the blend effect (Equation (3)) yields the formula for the loss modulus of the filled blend, see Equation (7) [12]:

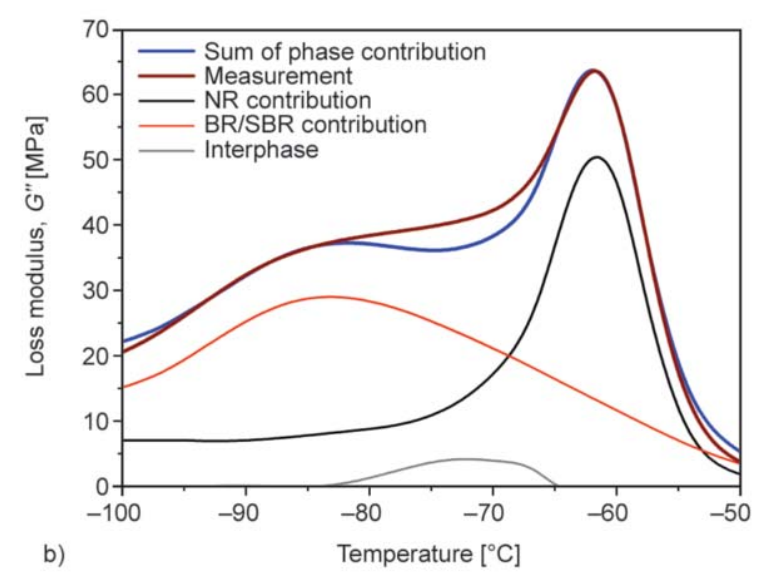

Figure 11. Separation of the unfilled NR/BR/SBR (70:15:15) (a) and (55:22.5:22.5) (b) blend into the contributions of the NR phase, the BR/SBR mixed phase and the interphase. 
$G_{\text {blend }}^{\prime \prime}=\sum_{\mathrm{i}}\left(1+\alpha_{\mathrm{i}} \frac{\phi_{\mathrm{i}}}{\phi_{\mathrm{F}, \mathrm{i}}+\phi_{\mathrm{i}}}\right) G_{\mathrm{P}, \mathrm{i}}^{\prime \prime}\left(a_{\mathrm{i}}+b_{\mathrm{i}}\left(\phi_{\mathrm{F}, \mathrm{i}}+\phi_{\mathrm{i}}\right)+c_{\mathrm{i}}\left(\phi_{\mathrm{F}, \mathrm{i}}+\phi_{\mathrm{i}}\right)^{2}\right)$

This equation allows estimating the peak heights of the polymer phases in blends when knowing the peak heights of the unfilled blend, the blend ratio of the polymers and the filler volume fractions in the polymer phases. Otherwise, the filler volume fractions in the polymer phases can be determined when the peak heights of filled and unfilled blends and the blend ratio are known. Therefore the unfilled blend is decomposed into the contributions of the different phases (Figure 11). The interphase is obtained as a difference between the measurement and the sum of phase contributions between both polymer phases.

The filled NR/BR/SBR blends are displayed in Figure 12. In the filled blends the filler increases the loss modulus of the phase in which the filler is located. For the NR/BR/SBR blends all filler is ascribed to the NR phase as the peak heights in the BR/SBR mixed-phase and the interphase are not increasing due to the filling. The calculated filler amount in the NR coincides well with the filler amount mixed into the compounds. A problem occurring in these blends is the overlap of the different phase contributions which makes a clear separation difficult. In addition, the BR/SBR mixedphase itself is no pure polymer phase. Possibly differently composed mixed phases with distinct glass transitions and filler affinities could be assumed. The phases with higher glass transition, containing more SBR do then superimpose with the NR phase.

\section{Conclusions}

Polybutadiene with a high cis- 1,4 content is crystallizing at low temperatures. As the crystallization

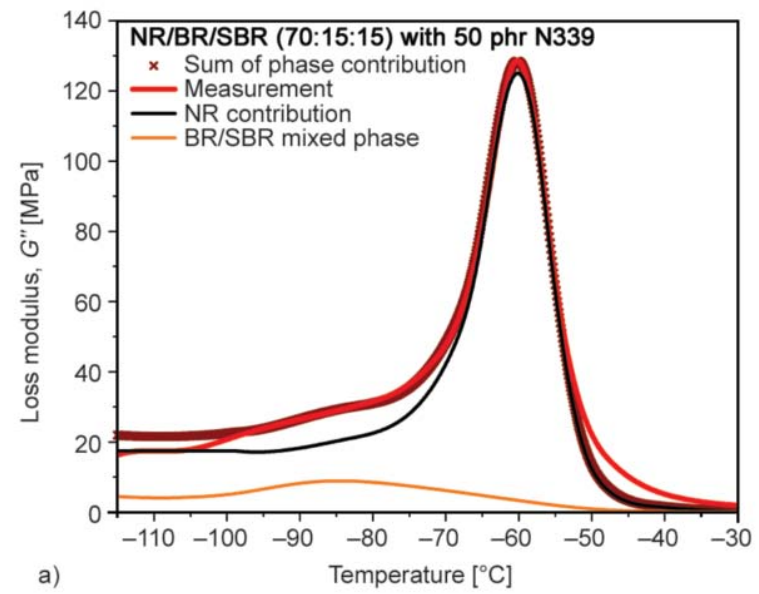

speed in a temperature range between -70 and $-40^{\circ} \mathrm{C}$ is sufficiently high, the crystallization can be seen in and it is influencing dynamic mechanical measurements. This effect depends on the measurement procedure, e.g. the temperature history of the sample and the heating rate. Filling with carbon black increases the effect of crystallization seen in the dynamic spectra. Blending with SBR which cannot crystallize is decreasing the effect. In this case BR and SBR are building a mixed phase.

The comparison of the dynamic spectra of unfilled and filled blends can yield the filler distribution in different phases of a blend. For NR/BR/SBR blends the filler seems to be located in the NR only. Thereby should be considered that due to the broadness of the $\mathrm{BR} / \mathrm{SBR}$ mixed peak a clear separation of the phase contributions is not guaranteed.

\section{Acknowledgements}

We would like to thank the Deutsche Forschungsgemeinschaft (DFG) for financial support (grant KL1409/9-1) and our project partners IPF Dresden and Continental AG for the very good cooperation.

\section{References}

[1] Eisele U.: Introduction to polymer physics. Springer, Berlin (1990).

[2] Bekkedahl N., Wood L. A.: Crystallization of vulcanized rubber. Industrial and Engineering Chemistry, 33, 381-384 (1941).

https://doi.org/10.1021/ie50375a023

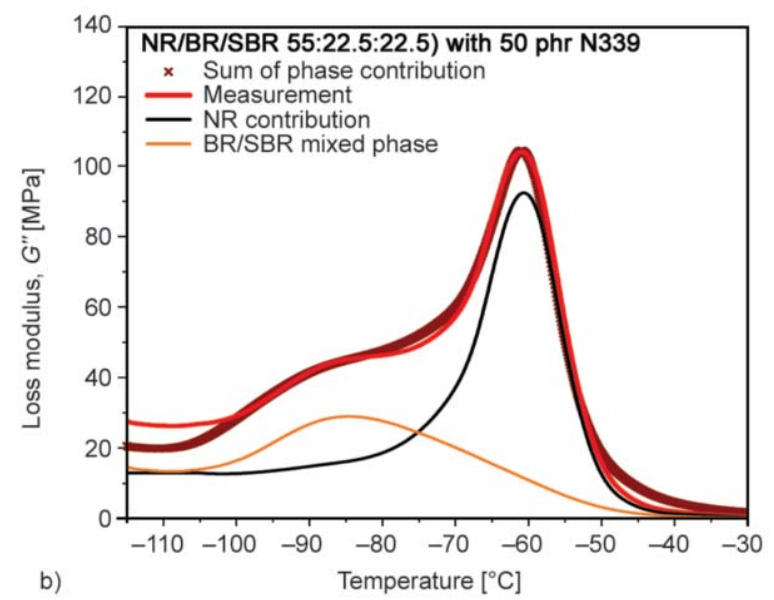

Figure 12. Separation of the filled NR/BR/SBR (70:15:15) (a) and (55:22.5:22.5) (b) blend into the contributions of the NR phase, the BR/SBR mixed phase and the interphase. Filler in the respective phases increases the peak amplitudes. 
[3] Bukhina K.: Low-temperature behaviour of elastomers. Koninklijke Brill, Leiden (2007).

https://doi.org/10.1201/b12239

[4] Wood L. A., Bekkedahl N.: Crystallization of unvulcanized rubber at different temperatures. Journal of Applied Physics, 17, 362-375 (1946). https://doi.org/10.1063/1.1707725

[5] Lion A., Johlitz M.: A thermodynamic approach to model the caloric properties of semicrystalline polymers. Continuum Mechanics and Thermodynamics, 28, 799-819 (2016). https://doi.org/10.1007/s00161-015-0415-8

[6] Sircar A. K., Lammond T. G.: Carbon black transfer in blends of cis-poly(butadiene) with other elastomers. Rubber Chemistry and Technology, 46, 178-191 (1973). https://doi.org/10.5254/1.3545009

[7] Schuster R. H.: Beziehung zwischen Verschnittmorphologie und physikalischen Eigenschaften von Elastomeren (in German). Gummi Fasern Kunststoffe, 49, 966-974 (1996).

[8] Morris M. C.: Rates of crystallization of cis-1,4-polybutadiene in elastomer blends. Rubber Chemistry and Technology, 40, 341-349 (1967). https://doi.org/10.5254/1.3539050

[9] Klüppel M., Schuster R. H., Schaper J.: Carbon black distribution in rubber blends: A dynamic-mechanical analysis. Rubber Chemistry and Technology, 72, 91108 (1999).

https://doi.org/10.5254/1.3538797

[10] Schuster R. H., Meier J., Klüppel M.: The role of interphase in filler partition in rubber blends. Kautschuk Gummi Kunststoffe, 53, 663-674 (2000).

[11] Meier J. G., Klüppel M., Geisler H., Schuster R. H.: Kieselsäuregefüllte Elastomerblends durch Masterbatchtechnologie (in German). Kautschuk Gummi Kunststoffe, 58, 587-594 (2005).

[12] Lorenz H., Steinhauser D., Klüppel M.: Morphology and micro-mechanics of filled elastomer blends: Impact on dynamic crack propagation. in 'Fracture mechanics and statistical mechanics of reinforced elastomeric blends' (eds.: Grellmann W., Heinrich G., Kaliske M., Klüppel M., Schneider K., Vilgis T.) Springer, Berlin, 81-128 (2013).

https://doi.org/10.1007/978-3-642-37910-9 3
[13] Wunde M., Klüppel M.: Influence of phase morphology and filler distribution in NR/BR and NR/SBR blends on fracture mechanical properties. Rubber Chemistry and Technology, 89, 588-607 (2016).

https://doi.org/10.5254/rct.16.83795

[14] Wunde M., Klüppel M.: Impact of mixing procedure on phase morphology and fracture mechanical properties of carbon black-filled NR/SBR blends. Continuum Mechanics and Thermodynamics, 29, 1135-1148 (2017). https://doi.org/10.1007/s00161-017-0562-1

[15] Strobl G.: Crystallization and melting of bulk polymers: New observations, conclusions and a thermodynamic scheme. Progress in Polymer Science, 31, 398-442 (2006).

https://doi.org/10.1016/j.progpolymsci.2006.01.001

[16] Rubinstein M., Colby R. H.: Polymer physics. Oxford University Press, New York (2003).

[17] Schuster R. H.: Verträglichkeit von Kautschuken in Verschnitten. Teil 1. Theorie und Methoden (in German). Wirtschaftsverband der deutschen Kautschukindustrie, Frankfurt (1992).

[18] Schuster R. H.: Verträglichkeit von Kautschuken. Ein altes und ein aktuelles Problem (in German). Die Angewandte Molekulare Chemie, 202, 159-185 (1992). https://doi.org/10.1002/apmc.1992.052020110

[19] Schuster R. H., Issel H. M., Peterseim V.: Selective interactions in elastomers, a base for compatibility and polymer-filler interactions. Rubber Chemistry and Technology, 69, 769-780 (1996). https://doi.org/10.5254/1.3538400

[20] Palierne J. F.: Linear rheology of viscoelastic emulsions with interfacial tension. Rheologica Acta, 29, 204-214 (1990). https://doi.org/10.1007/BF01331356

[21] Shi P., Schach R., Munch E., Montes H., Lequeux F.: Glass transition distribution in miscible polymer blends: From calorimetry to rheology. Macromolecules, 46, 3611-3620 (2013). https://doi.org/10.1021/ma400417f 